\title{
Philosophiques
}

\section{Présentation : Signification et portée des modèles d'évolution en économie et en sciences sociales}

\section{Jean Mathiot et Robert Nadeau}

Volume 25, numéro 2, automne 1998

Les modèles d'évolution en économie et en sciences sociales

URI : https://id.erudit.org/iderudit/027484ar

DOI : https://doi.org/10.7202/027484ar

Aller au sommaire du numéro

Éditeur(s)

Société de philosophie du Québec

ISSN

0316-2923 (imprimé)

1492-1391 (numérique)

Découvrir la revue

Citer ce document

Mathiot, J. \& Nadeau, R. (1998). Présentation : Signification et portée des

modèles d'évolution en économie et en sciences sociales. Philosophiques, 25(2),

151-161. https://doi.org/10.7202/027484ar d'utilisation que vous pouvez consulter en ligne. 
PHILOSOPHIQUES, VOL. XXV, $\mathrm{N}^{0}$ 2, AUTOMNE 1998, P. 151-161

ARTICLES

\title{
SIGNFICATION ET PORTÉE DES MODÈles D'ÉVOLUTION EN ÉCONOMIE ET EN SCIENCES SOCIALES
}

\section{PRÉSENTATION}

\author{
PAR \\ Jean Mathiot et Robert madead
}

Lors d'une journée d'études organisée le 26 février 1997 par le Séminaire d'épistémologie comparative du Département de philosophie de l'Université de Provence (Aix-Marseille 1), plusieurs chercheurs issus de disciplines différentes (économie, sociologie, philosophie) se sont brièvement retrouvés, le temps d'un débat argumenté (et présidé par Yves Duroux - CNRS, Paris) sur l'usage et la portée des modèles évolutionnaires - terme préféré ici pour sa neutralité à celui d'évolutionniste qui semble nous vouer à un choix de doctrine - dans les sciences sociales. C'est à l'ensemble de ces contributions sauf une, à savoir celle d'Alain Leroux, publiée indépendamment, que nous donnons accès dans le présent numéro thématique.

Pour avoir les uns et les autres eu à connaître de tels modèles dans leurs disciplines respectives, les participants sont venus afin d'interroger un usage effectif de tels modèles beaucoup plus que pour en établir à nouveaux frais les conditions. La question qui fit donc le lien entre tous fut à peu près celle-ci : que pense-t-on lorsqu'on introduit l'évolution dans un raisonnement économique ou sociologique?

Cette question a naturellement deux versants : que pense-t-on par rapport à ce qui fonctionne comme modèle, à savoir la théorie biologique de l'évolution, notamment dans la version darwinienne et néodarwinienne de la sélection naturelle? Mais aussi, en aval en 
quelque sorte, que pense-t-on comme effet de l'usage de ce modèle, et quelles transformations attendre dans la discipline d'accueil de cet usage?

Ces deux versants justifient le partage, un peu formel, des contributions à ce débat en deux demi-journées : la question critique adressée au modèle d'une part, l'analyse de ses effets et de ses justifications dans ses domaines d'importation d'autre part. Sans doute, les atouts reconnus à une rationalité évolutionnaire, et attribués à son terrain biologique d'origine, devraient se retrouver dans les effets. Il a semblé préférable de ne pas préjuger de ce rapport, au risque, peut-être, de laisser ouverte une question que ne manque pas de faire émerger la frustration de cette attente. Faut-il préciser que cette frustration est en fait apparue la règle plutôt que l'exception?

Du côté de la critique en amont, Maurice Lagueux (Université de Montréal) interroge le rapport entre sélection naturelle et rationalité : Pierre Livet (Université de Provence) met également à l'épreuve évolution et rationalité comme deux orientations proposées dans la théorie des jeux, et dont il compare les atouts respectifs, tandis que Jean Mathiot (Université de Provence) interroge les espoirs placés, à tort ou à raison, dans un modèle darwinien dévolution pour penser les phénomènes de convergence.

La théorie économique avec Alan Kirman, directeur d'études à l'École des Hautes Études en Sciences Sociales, la sociologie de Norbert Elias avec Roger Cornu (Université de Provence), la doctrine sociologico-économique de F. A. Hayek, enfin, avec Robert Nadeau (Université du Québec à Montréal) fournissent en aval des exemples privilégiés d'une épreuve par les effets.

Il était prévisible que le schématisme de ce programme initial dût éclater devant l'ampleur et la diversité des questions critiques. Car, après tout, il n'existe pas un modèle d'évolution préalablement assuré de son identité et de ses atouts, qui ne serait confronté qu'à des objets d'application. Lui-même est objet de discussion, de remise en question, comme on le voit avec les différentes versions de la postérité du darwinisme. Par ailleurs, l'usage de concepts évolutionnaires dans les différentes sciences sociales pose autant de questions lorsqu'il est revendiqué comme un succès (par exemple, chez Hayek ou chez Elias) que lorsqu'il semble entravé dans sa mise en ceuvre.

L'intérêt des contributions qu'on va lire tient précisément, semble-t-il, à la liberté que chacun a prise de poursuivre jusqu'au bout son questionnement initial, sans se préoccuper de se référer à un quelconque modèle standard de l'évolution ni à des critères prédéfinis de validité de l'usage qui en est fait.

Ce balayage des questions, loin d'aboutir à une dispersion, semble au contraire mieux expliciter ce qui est, dans des ouvrages plus spécialisés, maintenu à l'état latent. Pour ne prendre qu'un 
exemple, la distinction opérée par Alan Kirman entre une économie considérée comme un processus illimité dans le temps et dans ses transformations, et une économie considérée comme un système descriptible seulement à partir de l'analyse de ses états successifs d'équilibre, concerne la définition même de l'économie. L'usage de concepts évolutionnaires sert ici manifestement de révélateur d'enjeux qui dépassent la simple réflexion méthodologique. Ce genre de conclusion, que l'on trouvera également dans d'autres contributions, témoigne de la valeur de suggestion qui se dégage de cette liberté de ton: chacune des conclusions est comme une invitation à son approfondissement.

Si chaque contribution suggère ainsi au lecteur de poursuivre dans la direction qu'elle indique, l'ensemble témoigne de quelques fils conducteurs communs qui méritent d'autant plus d'être remarqués que rien ne les avait suggérés, et encore moins imposés, lors de l'organisation de ce débat.

Le premier de ces fils conducteurs paraît être une sorte de mise à distance de la biologie comme telle. Que l'enjeu de l'évolution ne s'identifie pas à l'usage d'un modèle spécifiquement biologique mérite d'être souligné. Historiquement d'abord : qu'il s'agisse de sociologie ou d'économie, le temps du modèle de l'organisme a vécu. Marshall illustre partiellement encore un tel souci en économique, de même que la pensée de la temporalité de l'organisme vivant hante Spengler ou Toynbee. Cette époque est révolue, et même, semble-til, depuis longtemps. Aujourd'hui, c'est à un autre niveau que se situe l'emprunt. Il ne paraît plus nécessaire d'assimiler la réalité sociale à la réalité organique pour pouvoir emprunter avec profit les schémas biologiques de l'évolution.

Sans doute la question renaît-elle comme question de la correspondance entre concepts biologiques (gènes, hérédité, mutations, environnement, spéciation, etc.) et concepts économiques ou sociologiques. Curieusement pourtant, cette question ne semble pas préalable. Elle surgit plutôt lorsqu'une première tentative d'user du modèle formel de l'évolution par sélection naturelle rencontre une limite. C'est au moment où la transposition devient nécessaire que surgit alors, paradoxalement, le problème de cette traduction. Mais ce moment de la transposition n'est pas premier ; il est plutôt le moment où justement s'impose à nouveau la différence d'objets, signe donc de la mise en défaut d'une communauté de problématique à laquelle le thème de l'évolution et de la sélection naturelle ouvre un droit sans barrière. La chose n'est pas étonnante si on se souvient qu'entre les sciences sociales et la biologie - prise en ce sens --, le commerce n'a jamais cessé dans les deux sens, ce que Hayek, du reste, met bien en évidence. Le darwinisme - et le concept de sélection naturelle - est ici assez naturellement le carrefour de cette circulation librement croisée. 
Ce qui caractérise néanmoins la période actuelle est le fait que, si nous admettons ce droit de libre circulation, nous sommes moins capables que par le passé de lui donner un contenu. De Botero à Benjamin Franklin, puis à Malthus et à Darwin, le thème de la population et du déséquilibre s'est déplacé d'un champ à l'autre en toute impunité et en toute transparence. En est-il de même pour l'évolution? Malgré les affirmations de F. Hayek ${ }^{1}$, qui croit voir dans l'évolution un thème issu des sciences sociales, l'originalité biologique du concept est à la fois incontestable et, pourtant, reste encore partiellement source d'énigmes. Nous acceptons certes l'idée de sa libre circulation entre les disciplines, mais nous ne sommes pas d'accord sur le contenu de ce qui circule.

D'oì un deuxième fil conducteur, venant guider et prolonger ce preınier pas qui nous libère d'une exigence d'analogie étroite avec la biologie. À un niveau plus élevé que celui d'une correspondance d'objets fonctionnerait un schéma de processus échappant à l'empire d'une construction rationnelle. L'acceptation de cette opposition massive entre, d'une part, un processus guidé par une visée rationnelle à la fois analytique et prédictive et, d'autre part, un processus réglé a posteriori par l'effet en retour de ses propres résultats, indépendamment de tout plan et de toute anticipation de ces résultats, semble assez largement partagée pour fédérer l'ensemble des réflexions sur le sens et l'intérêt des modèles évolutionnaires.

On peut concevoir cette économie de l'hypothèse de rationalité comme l'émergence d'un substitut inintentionnel des processus intentionnels. Dans le prolongement de cette démarche, on peut valoriser les avantages de cette substitution et espérer ainsi se décharger sur les mécanismes sélectifs de charges attribuées à la rationalité. La perspective hayékienne, et la théorie des jeux suivent précisément cette ligne.

1. Voir Droit, législation et liberté, une nouvelle formulation des principes libéraux de justice. et d'économie politique, Paris, PUF, vol. 1 : Règles et ordre, 1980, p. 26. Par ailleurs, Hayek est le premier à reconnaître que les sciences sociales, qui, comme c'est le cas patent de l'économique, sont aux prises avec des phénomènes complexes, ont de plus fortes affinités méthodologiques avec, en particulier, la biologie de l'évolution qu'avec la physique. Il écrit à ce propos : "(T)he existence of structures with which the theory of complexphenomena is concerned can be made intelligible only. by what the plysicists would call a cosmology, that is, a theory of their evolution. The problem of how galaxies or solar systems are formed and what is their resulting structure is much nore like the problems which the social sciences hase to face than the problems of mechanics; and for the understanding of the methodological problems of the social sciences a study of the procedures of geology or biology is therefore much more instructive than that of physics. "(F. A. Hayek, "Notes on the Evolution of Systems of Rules of Conduct [The Interplay between Rules of Individual Conduct and the Social Order of Actions] "dans Studies in Philosophy, Politics and Economics, Londres/Chicago, Routledge \& Kegan Paul/The University of Chicago Press, 1967, p. 76) Incidemment, la contribution de Robert Nadeau au présent numéro thématique foumit une analyse qui replace cette affirmation dans son contexte et vise à en elarifier la portée. 
Alors que cette approche est très largement reçue, on trouvera ici, sur des bases très diverses, voire opposées, des éléments critiques qui obligent à l'interroger. Non seulement les mécanismes sélectifs ne peuvent qu'artificiellement être mis en concurrence avec des procédés rationnels, mais il n'est pas sûr qu'ils aboutissent à des résultats semblables. Une évolution par sélection peut privilégier des résultats qu'aucune économie ou sociologie n'acceptera d'appeler optimaux ni même rationnels, comme le montrent l'économie industrielle ou même la théorie des jeux (voir les travaux de Weibull, souvent évoqués ici). On peut donc soupçonner que la rationalité doive déjà être présente en sous-main pour être retrouvée au terme d'une simple sélection. Ce soupçon, du reste, ne saurait épargner les concepts biologiques d'évolution eux-mêmes.

Du même coup, l'idée d'un relais avantageusement pris par une évolution de type sélectif sur une rationalité sociale a constructive " devient difficile à justifier. Ne se trompe-t-on pas de siècle et ne transpose-t-on pas abusivement à l'évolution les privilèges que l'empirisme anglais du dix-huitième siècle conférait à la nature sur la raison.? Le privilège de la nature pouvait alors s'analyser comme son opération invisible, agissante à un autre niveau que celui des résultats, mais aboutissant à des résultats semblables (finaux); l'évolution ne comporte aucunement cette facilité d'une causalité occulte mais substantielle, représentable comme agissant en sousmain et venant se substituer à la raison.

Faut-il dire pour autant que l'évolution sélective s'ajoute à la nature ? La question est débattue ici et tranchée en des sens différents selon les contributeurs, semble-t-il. De la thèse consensuelle que la sélection relève d'un type de mécanisme distinct d'une instruction déterministe (Darwin contre Lamarck), on peut en effet tirer deux conclusions inverses : soit la sélection vient en addition à un processus naturel, soit elle implique qu'on en bouleverse en totalité la conception. Dans les deux cas, on constate qu'on ne peut voir à l'œuvre deux procédés aboutissant au même résultat final (un optimum). La symétrie de l'intentionnel et de l'inintentionnel est toujours boîteuse quand il s'agit d'évolution.

En faveur de la seconde réponse, on peut noter que le rapport entre sélection et rationalité ne peut se limiter au thème de l'optimalité. On sait, d'une part, que celle-ci n'est pas un résultat à attendre inconditionnellement de l'évolution sélective; d'autre part, la sélection donne un sens à une autre version de la rationalité économique, plus globale celle-là : l'économie des coûts, c'est-à-dire des moyens'. La sélection étant par ailleurs gaspilleuse de la vie, c'est

2. C'est, en particulier, le sens ultime que Darwin donne à l'explication sélective de la fabrication cles cellules hexagonales des ruches chez les abeilles, ou encore des organes complexes, le bricolage de l'évolution récupérant tous les moyens disponibles et les rendant même récupérables, facilitant ainsi la genèse des fonctionnalités. 
à un remaniement global de la rationalité qu'il faudrait ainsi se résoudre pour comprendre cette nouvelle cohérence.

C'est donc, logiquement, un troisième ordre de questions qui sous-tend la critique et doit la mener éventuellement à une issue moins négative. S'il est vrai, par exemple, qu'en théorie des jeux, l'issue des changements "évolutifs" des stratégies dépend essentiellement de la structure qu'on s'est donnée au départ, il est flagrant que l'on retombe ainsi sur un mécanisme déterministe dont les conditions initiales redeviennent décisives pour le devenir du système considéré. L'application d'un modèle d'évolution tuerait donc ce qu'il y a d'indéterministe dans l'évolution - caractère pourtant essentiel dans la filiation darwinienne. C'est la même contradiction qui affecte la notion, paradoxale, de stratégie évolutionnairement stable de J. Maynard-Smith qui vient clôturer comme définitif, au nom de l'évolution, l'état d'un système.

Ces contradictions, soulignées par plusieurs des présentes contributions (ici encore, sans aucune concertation et, répétons-le, sur des bases interprétatives fort différentes), sont peut-être le troisième fil conducteur que l'on trouvera ici pour une investigation sur l'usage des modèles évolutionnaires. Tiennent-elles à la simple imperfection qui s'attacherait au principe même d'une mise en ouvre d'un modèle en raison du péché originel de l'emprunt? Rien ne semble ici confirmer une telle hypothèse. C'est plutôt que lon ne prend pas le problème d'assez haut. C'est, au contraire, à l'approfondissement d'une nouvelle articulation entre ce qu'on peut appeler un horizon indéterministe et un jeu de déterminations dans le temps qu’il faut procéder. Ici encore, la biologie peut être invoquée : mais c'est moins comme archétype que comme témoin l'un problème épistémologique tout simplement commun aux sciences concernées. Si l'évolution n'est plus aux frontières de la connaissance, selon le mot de Jacques Monod, c'est que le jeu du hasard et de l'imprévisible doit être réintégré au cœur de la connaissance. On a bien, alors, un problème épistémologique général à résoudre, dont les difficultés ne tiennent plus à des phénomènes de transfert locaux entre domaines d'objets différents ${ }^{3}$.

3. Cette discussion de l'enjeu épistémologique - et non théorique au sens étroit lié à un domaine d'objets - de l'évolution importe, car si on peut en conclure que l'opération de traduction entre le biologique et l'économique n'est pas une opération pertinente pour capter cet enjeu, on peut même aller plus loin en soutenant que celte traduction, si on la prenait comme un préalable, n'aurait que des effets négatifs en assinilant ce qui ne doit pas l'être. Car, dans ce cas, la traduction ou la correspondance n'est alors plus porteuse de ce qui est utilement commun aux deux problématiques. Elle n'est plus qu'une assimilation fautive d'objets différents. Chercher, par exemple, à tout prix à quoi correspondent donc en économie les gènes de la biologie risque alors de n'être qu'une fausse piste. Cette remarque a son importance, notamment pour la théorie des jeux qui tente une mise en correspondance rigoureuse de concepts comme ceux de reproduction, mutations, etc. avec ses propres concepts. 
Les remarques précédentes ne visent qu'à introduire à la lecture des contributions qui suivent et que l'on résumera maintenant à grands traits en terminant cette introduction. Maurice Lagueux, dans sa contribution intitulée "Rationalité et sélection naturelle en économie ", pose précisément la question de savoir à partir de quel moment on peut considérer une explication comme spécifiquement darwinienne, c'est-à-dire spécifiquement sélective. Si les feuilles d'un arbre poussent régulièrement sur son pourtour du lait que le Soleil est le facteur de leur croissance, pour reprendre un exemple fameux de Milton Friedman, faut-il parler d'adaptation des feuilles et de répartition optimalisée par sélection ? En répondant par la négative, Maurice Lagueux montre l'incompatibilité des deux séries explicatives et, selon lui, l'impropriété de la seconde lorsque la première est possible. Il ouvre donc la question cruciale de l'extériorité des deux séries ; une conséquence s'en dégage immédiatement, qui oblige à contester la symétrie que Milton Friedman croyait pouvoir tirer de son exemple, entre un modèle intentionnel de croissance active des feuilles et un modèle inintentionnel d'adaptation passive à l'exposition au Soleil. La symétrie serait doublement fautive : d'une part, le processus de croissance n'a rien qui soit assimilable à de l'intentionnel ; d'autre part, il n'aurait rien non plus d'une adaptation passive par sélection. En concluant ainsi qué sélection et processus déterministe sont réellement hétérogènes, Maurice Lagueux établit l'irréductible spécificité d'un argument sélectif, et ainsi l'impossibilité de le penser' sur la base d'une identité de résultats avec d'autres processus. En économie, l'argument sélectif ne saurait donc concerner, selon Maurice Lagueux, que l'irrationnel que comportent les processus auxquels participent les acteurs économiques.

C'est aussi, en définitive, à l'échec d'une substitution de l'argument sélectif à un argument par la rationalité que conclut Pierre Livet, au terme d'une analyse des ambitions de la théorie des jeux évolutionnaires dans sa contribution intitulée a Jeux évolutionnaires et paradoxe de l'induction rétrograde (backward induction) ". Un jeu évolutionnaire au sens moderne étudie des populations de joucurs dotés d'une stratégie ou d'un ensemble de stratégies qui sont des programmes, à la différence de la version raisonneuse qui donne aux joueurs la faculté d'anticiper sur les stratégies des autres joneurs et de régler la formation de leur stratégie sur le résultat de l'emboîtement des interactions virtuelles qu'ils imaginent : ainsi en est-il dans le raisonnement rétrograde. Binmore et les partisans des jeux évolutionnaires pensent tout d'abord obtenir de la logique évolutionnaire un mode d'accès aux équilibres de Nash (qui, comme on sait, ne comportent pas en eux-mêmes l'indication du chemin qui y mène); ils espèrent aussi retrouver les résultats de la version raisonneuse sans avoir besoin de créditer les joueurs de capacités cognitives complexes et irréalistes. En outre, ils pensent dépasser le modèle des équilibres de Nash et échapper aux paradoxes des 
résultats des équilibres épistémiques produits par les raisonnements en boucle de la backward induction. Sur tous ces points, Pierre Livet montre que ces espoirs sont exagérés. L'approche sélective n'obtient, en effet, les résultats invoqués qu'en fonction de la dynamique réplicative choisie au départ. De plus, il n'y a pas nécessairement coïncidence entre les stratégies évolutionnairement stables et les stratégies sélectionnées.

Élargissant le propos, Pierre Livet suggère une comparaison systématique entre une approche évolutionnaire et une approche par équilibres interactifs, permettant d'aller au-delà des équilibres de Nash par le jeu des révisions virtuelles imaginées par les agents. Sans doute a-t-on quitté alors les raisonnements à partir des informations prises comme données. De telles révisions actionnelles mettent assurément en jeu une nouvelle forme d'incertitude sur le contexte de l'action. Mais Pierre Livet propose justement de comparer cette incertitude avec celle que tout modèle d'évolution met en ceuvre. Selon ce point de vue, un modèle évolutionnaire ne mobiliserait pas moins que l'approche par la rationalité interactive l'incertitude du contexte. Il aurait, en revanche, l'inconvénient de ne pas en faire bénéficier les joueurs. Ainsi peut s'inverser, en quelque sorte, le privilège revendiqué par l'approche évolutionnaire dans le cadre de la théorie des jeux par rapport à leur version raisonneuse.

La contribution de Jean Mathiot, "Évolution, sélection, information. La question de la convergence ", reprend sous une autre forme la question de l'hétérogénéité de l'évolution sélectionnante darwinienne par rapport à la représentation de la convergence d'un processus. On ne peut y voir un processus convergeant sur un optimum. Les trois termes manquent : le critère (l'utilité) n'est jamais fixé, de sorte que l'optimalité n'est jamais repérable comparativement, encore moins comme principe de choix; la convergence n'a pas de sens comme terme d'aboutissement et ce qui invalide la notion de stratégie évolutionnairement stable; la notion de processus même, enfin, est mise à mal dans le schéma darwinien. Cela, bien entendu, invalide aussi des cadres comme la théorie des jeux pour servir d'espace d'interprétation d'un modèle d'évolution. Ce diagnostic critique n'est pourtant pas négatif : dans l'évolution darwinienne se joue, en effet, une appréhension de l'événement que l'on doit penser en termes d'information, et non de changement dans un état ou par rapport à un état (c'est-à-dire comme processus). C'est là, si l'on veut, une nouvelle acception de la notion de convergence. Autant dire qu'il s'agit d'une révolution épistémologique, et pas seulement théorique, qui peut intéresser, par exemple, le statut des anticipations en économie.

Comme on l'a déjà fait remarquer, les contributeurs de la seconde demi-journée n'ont pas moins posé des problèmes fondamentaux, en dépit de l'aspect plus “ appliqué » des objets de leurs interventions. Ainsi, Alan Kirman, dans sa contribution intitulée " Évolution et 
théorie économique ", part du conflit, tel que la théorie économique le reflète en elle-même, entre une approche en termes d'équilibre, dont l'évolution est absente, et une approche évolutionnaire. Ce conflit, montre-t-il, n'est nullement résorbé actuellement par les divers moyens mis en ouvre pour appliquer un modèle d'évolution. Mutations aléatoires, environnement, aptitude, reproduction, spéciation sont autant de pierres d'achoppement à une réelle correspondance entre le biologique évolutionniste et l'économie. On remarquera que les problèmes posés alors doivent aussi être renvoyés à la biologie elle-même : ils ne tiennent pas à la seule « application ". Au total, dans une sorte de position de repli, on peut penser, avec Kirman, que les théoriciens de l'économie ont tenté d'utiliser l'évolution comme un moyen de sélectionner parmi des stratégies existantes plutôt que comme un moyen de dépeindre un monde illimité se développant. Et l'analyse des jeux évolutionnaires, auxquels cet auteur, en spécialiste, reconnaît le mérite d'une mise en correspondance plus rigoureuse des termes, fait apparaître le retour à des modèles déterministes ou stochastiques dans lesquels la structure de départ redevient décisive pour déterminer la dynamique, en contradiction avec la logique même de l'évolution. Malgré cette constante dominance de l'approche standard en termes d'équilibre sur une approche qui ferait en quelque sorte de l'économie un système en perpétuel développement dans lequel l'environnement et la nature des acteurs seraient sujets au changement, Alan Kirman conclut à la nécessité de poursuivre l'exploration de la seconde voie.

C'est aussi sur un fond d'incompréhension toujours possible et de risque de malentendu que Norbert Elias développe sa sociologie évolutionniste. Dans sa contribution, qui a pour titre a Évolution et processus configurationnel chez Norbert Elias ", Roger Cornu montre comment la notion de configuration est chargée par N. Elias de rompre avec l'usage traditionnel de l'évolution pour penser l'intégration sociale comme phénomène global. Ce concept doit rompre avec la fausse alternative d'une intégration globale ou d'une intégration individuelle de l'individu dans le social : ni Durkheim ni Tarde. L'évolution ainsi remodelée conduit N. Elias à insister sur le rôle de l'héritage des conditions de l'action des individus, et sur les liens d'interdépendance et d'interpénétration qui se jouent entre les individus, au niveau de leur pratique. Selon trois niveaux (développement technologique, rapports sociaux, processus de civilisation) se définit de la sorte un champ de tension et de conflits de pouvoir. Le thème évolutionnaire consiste, alors, à analyser les déformations des configurations ainsi décrites, déformations qui prennent la forme de processus compréhensibles et accessibles à la pratique même des individus. L'évolution donne accès pour le théoricien à des concepts structurels proprement sociologiques, dissociables des concepts d'expérience liés à la simple chronologie, comme la sociologie se dissocie de l'histoire. Il est à noter que l'évolution fonctionne comme un schéma d'interprétation que 
N. Elias considère efficace dans la situation qui est selon lui la nôtre, et dans laquelle les faits pertinents ne manquent pas, mais sont au contraire en profusion.

On conviendra aisément, enfin, que la figure de Friedrich Hayek est difficilement contournable pour qui étudie la place et la portée des modèles d'évolution en sciences sociales, et en particulier en économie. Dans son analyse, “ L'évolutionnisme économique de Friedrich Hayek ", Robert Nadeau entreprend de tirer au clair les tenants et aboutissants de la perspective évolutionnaire que ce prix Nobel d'économique a fait sienne. Les idées de Hayek, faut-il le rappeler, sont revenues à l'avant-scène depuis la défaillance des politiques économiques interventionnistes inspirées des théories keynésiennes. Or, l'examen de la doctrine hayékienne permet d'apercevoir clairement qu'il y a un lien entre le renouveau du libéralisme, comme philosophie économique et sociale (qui conduit directement au rejet de l'État-providence), et l'adoption d'une perspective évolutionnaire, tout comme il y a également un lien serré chez cet économiste entre la critique du socialisme - peut-être le leitmotiv central de l'ouvre de Hayek - et l'insistance sur limportance des ordres spontanés.

Robert Nadeau fait voir comment Hayek pense pouvoir fonder la supériorité de l'économie de marché, pensée justement comme ordre social spontané, sur l'économie planifiée. D'abord, Hayek retient de Michael Polanyi que l'activité humaine est en grande partie basée sur une " connaissance tacite ", qui ne s'acquiert que localement et par imitation, et qui ne saurait être emmagasinée dans un seul et unique cerveau qui servirait de centre de décision et de coordination des agents économiques individuels. Ce qui, suivant Hayek, fait l'efficacité supérieure de l'économie de marché, c'est précisément qu'elle tire mieux parti de l'immense quantité de connaissances dispersées entre les individus : l'ordre économique spontané est, ainsi, en très grande partie fonction de la compétence épistémique des individus. Mais il y a plus. Car, pour Hayek, si, en tant qu'agents économiques, les individus parviennent à se coordonner entre eux par l'intermédiaire du mécanisme des prix, c'est aussi, plus fondamentalement, parce qu'ils suivent inconsciemment les règles de conduite et de perception que leur culture traditionnelle leur a inculquées. Ces règles, dont Hayek n'hésite pas à dire qu'elles ne sont pas du tout de la même nature que les préceptes éthiques que la raison philosophique voudrait imposer, sont économiquement déterminantes parce qu'elles assurent la survie des individus dans leur groupe d'appartenance, et aussi parce qu'elles permettent aux groupes qui les suivent d'être favorisés dans la concurrence économique et donc, ultimement, d'être, à proprement parler, sélectionnés culturellement. Si c'est le cas, insiste Robert Nadeau, alors l'ordre économique spontané doit être aussi vu comme dépendant de la compétence morale des individus. 
Ainsi, Hayek relie causalement la libre coordination économique et sociale que rend possible l'économie de marché, ce qu'il préfère appeler la catallaxie, et le plus grand degré d'adaptation économique des groupes humains en compétition les uns avec les autres, ellemême fonction de la tradition culturelle propre à ces divers groupes, dont les plus dynamiques se développent au détriment des moins efficaces. C'est une fois examinée minutieusement cette articulation que deviennent plus manifestes le sens et la portée de l'évolutionnisme économique endossé par Hayek. Suivant l'analyse de Robert Nadeau, ce qui caractérise la perspective hayékienne comme étant radicalement évolutionnaire, c'est qu'elle se définit strictement dans une optique populationnelle. Hayek redécouvre, en effet, les théorèmes de Thomas Malthus et, en montrant pourquoi ces théorèmes ne sont plus, comme tels, applicables à la situation économique qui prévaut dans les sociétés industrielles avancées et en les modifiant en conséquence, il en vient à conclure que ce qui doit nous convaincre de la supériorité évolutionnaire de la catallaxie, c’est qu'elle permet à plus d'individus de naître, de se nourrir et de se reproduire. C'est dans ces termes qu'il faut comprendre la conjecture hayékienne de la sélection de groupe. Ce ne serait donc pas tant parce qu'elle permettrait d'atteindre une population planétaire de taille optimale, mais plutôt parce qu'elle permettrait à la sélection économique des individus et des groupes de se faire spontanément jour, et parce qu'elle rendrait possible que le processus de coordination économique se poursuive indéfiniment en permettant aux groupes économiques dominants de croître aux dépens des autres, que l'économie de marché serait présentée par Hayek comme évolutionnairement supérieure à toute forme d'économie basée sur' l'intervention de l'État.

Quelles que soient les orientations des diverses contributions, dont la liberté de propos était, répétons-le, la règle du délbat, il ressort que le thème évolutionnaire n'est que rarement invoqué sans soulever l'image, le regret ou la revendication d'un conflit. Thème critique done, il ouvre un chantier qui déborde évidemment la notion de simple exportation d'un modèle : la ligne de partage qui l'accompagne n'épargne, semble-t-il, ni la discipline d'accueil (sociologie ou économique) ni la biologie elle-même, souvent interrogée ici dans ses propres variantes, Ouvrir et baliser ce chantier critique serait le meilleur prolongement de ce débat.

Département de philosophie

Université de Provence.
Département de philosophie

Université du Québec à Montréal 